\title{
La formation des cadres éducatifs : quelle dimension internationale?
}

\section{Bernard Dizambourg}

\section{OpenEdition}

1 Journals

Édition électronique

URL : https://journals.openedition.org/ries/1215

DOI : 10.4000/ries. 1215

ISSN : 2261-4265

Éditeur

France Education international

\section{Édition imprimée}

Date de publication : 1 décembre 2005

Pagination : 21-23

ISSN : 1254-4590

\section{Référence électronique}

Bernard Dizambourg, «La formation des cadres éducatifs : quelle dimension internationale? », Revue internationale d'éducation de Sèvres [En ligne], 40 I décembre 2005, mis en ligne le 10 avril 2012, consulté le 05 juillet 2021. URL : http://journals.openedition.org/ries/1215 ; DOI : https://doi.org/ $10.4000 /$ ries. 1215 


\section{La formation des cadres éducatifs : quelle dimension internationale?}

\section{Bernard Dizambourg}

La nécessité de former les cadres des institutions éducatives ne semble aujourd'hui plus faire débat, bien que ceux qui accèdent à ces fonctions présentent déjà une formation antérieure de niveau élevé. Mais la spécificité de ces métiers comparativement à ceux préalablement exercés, souvent celui d'enseignant, justifie la nécessité d'une formation initiale et continue de ces cadres.

Constatons que la formation de ces cadres ne laisse que très peu de place à la dimension internationale, en France comme souvent à l'étranger, en particulier en Europe. Notons aussi que cette dimension fait l'objet d'assez peu de demandes de la part des cadres eux-mêmes. Ainsi, une enquête récente de la direction des Études et de la prospective du ministère de l'Éducation nationale soulignait la satisfaction de chefs d'établissements à l'égard de la formation, en particulier les dimensions échanges d'expérience et analyse de pratiques, évoquait la demande de formation complémentaire dans le domaine juridique et financier. La dimension internationale apparaissait assez peu présente dans les attentes des chefs d'établissements interrogés.

Pourtant dans une évaluation réalisée par l'École supérieure de l'Éducation nationale (ESEN) auprès de cinquante stagiaires ayant participé au séminaire européen de 2004, $84 \%$ des participants reconnaissent l'intérêt d'un tel séminaire et précisent à $70 \%$ que les thèmes traités dans le séminaire sont de nature à construire et à renforcer des compétences. $48 \%$ pensent, en outre, que les acquis de la semaine auront une incidence sur leurs pratiques professionnelles. Parmi les thématiques proposées, celle du croisement de regard sur les systèmes éducatifs européens recueille $84 \%$ de satisfaction devant celle de la comparaison du fonctionnement et de l'évaluation des établissements scolaires (74\%).

Au vu de ce constat, une question doit être posée : est-il souhaitable de prévoir une dimension internationale dans la formation des cadres des institutions de formation, en particulier des cadres des systèmes scolaires? Une dimension comparatiste au niveau international, et en particulier européen, participe-t-elle à la prise de recul nécessaire à tout cadre par rapport à la pratique de terrain?

La réponse à cette question doit prendre en considération les conclusions de nombreuses études et recherches : les systèmes éducatifs des pays développés sont confrontés aujourd'hui à une assez grande similitude d'enjeux. Identifions trois traits de convergence principaux. 
On constate une attente de plus en plus forte à l'égard des systèmes éducatifs, l'élévation des compétences et du niveau de formation étant conçue comme la réponse première au contexte de compétition accrue liée à la globalisation économique. Cette dernière se traduit à la fois par une exigence de performance vis-à-vis des institutions de la part des autorités de tutelle mais aussi sous forme d'une demande de réussite scolaire de la part des familles au profit de leur enfant, demande s'exprimant fréquemment par une montée du consumérisme scolaire.

La fragmentation du milieu social se traduit par une hétérogénéité croissante des situations d'environnement de l'institution scolaire : hétérogénéité des territoires, segmentation sociale accrue des espaces urbains et ruraux, tendance au repli communautaire sont le lot commun de nombreux systèmes éducatifs. Ajoutons que le tassement démographique commun à de nombreux pays accentue les concurrences entre territoires et rend plus complexe la recherche de l'équilibre entre différenciation des réponses éducatives face à ces segmentations des situations et égalité de traitement.

Enfin, le malaise enseignant est une situation très fréquemment identifiée, de nature différente selon les pays : parfois interrogation sur les missions (enseigner? former? éduquer?) et la confrontation de celles-ci avec les contextes évoqués précédemment, ailleurs expression d'un stress ressenti attribué à la régulation par les résultats. Là aussi, dans de nombreux pays, en particulier européen, enseigner est désormais perçu par les enseignants eux-mêmes comme un métier difficile.

Si l'environnement de nombreux systèmes éducatifs présente d'assez fortes similitudes, il nous semble aussi intéressant de regarder les points de convergence ou de divergence sur trois déterminants des modes de régulation des systèmes éducatifs qui définissent assez lourdement le rôle du cadre:

- la relation entre le cadre (le cadre pédagogique plus particulièrement) et l'enseignant, avec l'interrogation sur le comment favoriser la capacité des cadres pédagogiques à agir sur les enseignants et développer ainsi la mise en cohérence de l'action pédagogique; sur cette question, si de nombreux pays considèrent que l'exercice préalable d'une fonction d'enseignant (cas le plus général) est une condition nécessaire, le clivage réside beaucoup plus sur la façon de réaliser le passage d'une fonction à une autre : en organisant un passage progressif et dans la continuité du premier métier (comme le système éducatif anglais le fait) ou en marquant la rupture entre les deux fonctions, comme le mode de recrutement des cadres en France l'organise plus nettement;

- la relation entre les institutions éducatives et la régulation politique : constatons que cette dernière fait l'objet dans de très nombreux pays d'une régulation de plus en plus partagée entre autorités centrales et autorités politiques décentralisées, avec un fort investissement du niveau central sur les fonctions de pilotage et d'évaluation et une prise en charge plus ou moins forte d'un niveau 
de gestion par le niveau décentralisé; cette situation appelle sans aucun doute une grande capacité des cadres, en particulier des chefs d'établissements, à maîtriser des fonctions de médiation complexe entre les différents niveaux d'autorité politique et l'établissement;

- la dynamique de l'évaluation est considérée dans de très nombreux pays comme étant le processus devant permettre de relier l'impulsion centrale et la responsabilisation des établissements; constatons cependant une forte différence entre les situations où l'évaluation est d'abord externe à l'établissement et celles qui fondent le processus avant tout sur des dispositifs d'autoévaluation de l'établissement précédant une évaluation externe.

L'action de formation est significativement enrichie lorsque que l'on permet aux cadres de comparer leurs pratiques sur ces différents aspects, mettant ainsi en évidence à la fois des similitudes d'enjeux et des modes de réponse parfois très différenciées. Tout cadre doit d'assurer dans sa mission une part de prise en charge de la transformation de notre système éducatif et c'est bien ce niveau de compétence que l'on cherche alors à consolider.

C'est dans cette direction que l'ESEN a décidé de développer son action au moyen de plusieurs modalités :

- organisation en formation initiale et en formation continue d'un séminaire européen annuel permettant à nos publics de mieux connaître les systèmes éducatifs de nos partenaires;

- introduction dans de nombreux séminaires thématiques d'une dimension comparatiste par la présence d'intervenants étrangers

- constitution d'un réseau européen d'écoles de formation de cadres devant permettre dans un premier temps travail commun et échanges de formateurs, dans un second temps actions communes de formation vers les cadres.

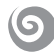

Travailler la dimension internationale de la formation peut parfois être considéré comme relevant d'un supplément plus ou moins optionnel du «noyau dur» de la préparation du cadre. Pour l'ESEN, cette dimension de formation contribue pleinement à l'acquisition et au développement des compétences nécessaires à l'exercice professionnel. C'est aussi cet objectif qui légitime le travail commun de l'ESEN avec le Centre international d'études pédagogiques et la Revue internationale d'éducation de Sèvres. 
